\title{
STRATIFICATION AND COORDINATE SYSTEMS FOR THE MODULI SPACE OF RATIONAL FUNCTIONS
}

\author{
MASAYO FUJIMURA AND MASAHIKO TANIGUCHI
}

\begin{abstract}
In this note, we give a new simple system of global parameters on the moduli space of rational functions, and clarify the relation to the parameters indicating location of fixed points and the indices at them. As a byproduct, we solve a conjecture of Milnor affirmatively.
\end{abstract}

\section{INTRODUCTION}

Let Rat ${ }_{d}$ be the set of all rational functions of degree $d>1$, and $\mathrm{M}_{d}$ the set of all Möbius conjugacy classes of elements in Rat ${ }_{d}$, which is called the moduli space of rational functions of degree $d$.

Here it is a fundamental problem to give a good system of parameters on $\mathrm{M}_{d}$. And McMullen showed in [5] that, outside the Latté loci, every multiplier spectrum at periodic points corresponds to a finite number of points in $\mathrm{M}_{d}$. This result is epoch-making, and many studies have been done on the system of multipliers, or indices, at periodic points. Among other things, the following example is well known.

Example 1.1 (see [6]). When $d=2$, there are 3 fixed points counted including multiplicities, the indices of which satisfy a single simple relation, called Fatou's index formula. Hence, we can consider a map $\Phi_{2}: \mathrm{M}_{2} \rightarrow \mathbb{C}^{2}$ induced by two of three fundamental symmetric functions of multipliers at fixed points. This map $\Phi_{2}$ is bijective, and hence gives a coordinate system for $\mathrm{M}_{2}$.

Remark 1.2. In the case of polynomials, the set of multipliers, or indices, at fixed points gives an interesting system of parameters on the moduli space of polynomials. For the details, see [3] and [4].

Clearly, the multipliers at fixed points only are not enough to parametrize the moduli space $\mathrm{M}_{d}$ when $d>2$. But, it seems difficult to find a suitable set of multipliers at periodic points for obtaining a good system of global parameters. On the other hand, in the case of polynomials, the set of monic centered ones is often used as a virtual set of representatives of points in the moduli space MPoly ${ }_{d}$ of polynomials of degree $d$, and it is well known that the coefficients of them give a useful set of parameters on MPoly $_{d}$, which in particular induces the complex orbifold structure of $\mathrm{MPoly}_{d}$. We give, in $\S 2$, a family of rational functions whose

Received by the editors August 17, 2009.

2010 Mathematics Subject Classification. Primary 30C15; Secondary 37F10.

The second author is partially supported by Grant-in-Aid for Scientific Research (C) 19540181.

(C)2010 American Mathematical Society Reverts to public domain 28 years from publication 
coefficients give a good system of parameters on $\mathrm{M}_{d}$ in a similar sense as in the case of the family of monic centered polynomials.

In $\S 3$, we investigate the correspondence between these coefficient parameters and the union of the set of the indices and location of fixed points, which gives a candidate of an important subsystem of parameters on $\mathrm{M}_{d}$. Here, the overlap type of fixed points naturally gives a stratification of $\mathrm{M}_{d}$. We introduce a natural system of coordinates on each stratum. As a byproduct, we give an affirmative answer to a conjecture of Milnor proposed in the book [7].

\section{A NORMALIZED FAMILY OF RATIONAL FUNCTIONS}

A general form of a rational function of degree $d$ is

$$
\frac{P(z)}{Q(z)}
$$

with polynomials $P(z)$ and $Q(z)$ of degree at most $d$, where $P(z)$ and $Q(z)$ have no common non-constant factors and one of them has $d$ as the degree. To consider the moduli space $\mathrm{M}_{d}$, we may assume without loss of generality that $Q(z)$ is of degree $d$, and that the resultant $\operatorname{Resul}(P, Q)$ of $P(z)$ and $Q(z)$ does not vanish. Also it imposes no restriction to assume that $Q(z)$ is monic. We call such a rational function satisfying the above conditions a canonical function.

Definition 2.1. The canonical family $C_{d}$ of rational functions of degree $d$ is defined as the totality of canonical functions of degree $d$ as above:

$$
\left\{R(z)=\frac{P(z)}{Q(z)} \in \operatorname{Rat}_{d} \mid \operatorname{deg} Q=d, \operatorname{Resul}(P, Q) \neq 0, Q \text { is monic }\right\} .
$$

Moreover, writing

$$
P(z)=a_{d} z^{d}+\cdots+a_{0}, \quad Q(z)=z^{d}+b_{d-1} z^{d-1}+\cdots+b_{0},
$$

we call the vector $\left(a_{d}, \cdots, a_{0}, b_{d-1}, \cdots, b_{0}\right)$ the system of coefficient parameters for $C_{d}$.

Every point in $\mathrm{M}_{d}$ contains an element in $C_{d}$ as a representative. On the other hand, since $\mathrm{M}_{d}$ is $(2 d-2)$-dimensional, while the dimension of $C_{d}$ is $2 d+1$, we can consider three normalization conditions on elements in $C_{d}$. Here we impose

$$
a_{0}=0, \quad b_{1}=-1, \text { and } b_{0}=1 .
$$

We call a rational function in $C_{d}$ satisfying these conditions a normalized function.

Definition 2.2. We call the family consisting of all normalized functions in $C_{d}$ the normalized family of degree $d$, which is denoted by $N_{d}$.

More explicitly,

$$
N_{d}=\left\{\frac{a_{d} z^{d}+\cdots+a_{1} z}{z^{d}+b_{d-1} z^{d-1}+\cdots+b_{2} z^{2}-z+1} \in C_{d}\right\},
$$

and we call the vector $\left(a_{d}, \cdots, a_{1}, b_{d-1}, \cdots, b_{2}\right)$ the system of coefficient parameters for $N_{d}$. Here, we can show that $N_{d}$ is an ample family of rational functions for every $d$. 
Example 2.3. When $d=2$, the natural projection of $N_{2}$ to $\mathrm{M}_{2}$ is surjective. To see this, it suffices to show that every possible set of multipliers $\left\{m_{1}, m_{2}, m_{3}\right\}$ at fixed points corresponds to a rational function in $N_{2}$ (cf. Example 1.1).

First, if the set is $\{1,1,1\}$, then a corresponding rational function in $N_{2}$ is uniquely determined (cf. Example 3.5) and is

$$
R(z)=\frac{-z^{2}+z}{z^{2}-z+1}
$$

If the set is $\{1,1, m\}$ with $m \neq 1$, then a corresponding rational function is

$$
R(z)=\frac{z(m z+p)}{p\left(z^{2}-z+1\right)}
$$

with a solution $p$ of $p^{2}+(m+1) p+m^{2}=0$.

Next, in the remaining cases, the set $\left\{m_{1}, m_{2}, m_{3}\right\}$ of multipliers satisfies that $m_{j} \neq 1(j=1,2,3)$ and Fatou's index formula

$$
\frac{1}{1-m_{1}}+\frac{1}{1-m_{2}}+\frac{1}{1-m_{3}}=1
$$

Here if the set is $\{0,0,2\}$, we can see that a corresponding rational function is

$$
R(z)=\frac{(3 / 2) z^{2}}{z^{2}-z+1}
$$

And otherwise, we can choose $m$ and $m^{\prime}$ among $\left\{m_{1}, m_{2}, m_{3}\right\}$ so that

$$
m^{\prime} \notin\{0, \pm i / \sqrt{3}\}, m m^{\prime}-1 \neq 0, \text { and } m+m^{\prime}-2 \neq 0,
$$

which are assumed to be $m_{1}$ and $m_{2}$, respectively. Then the equation

$$
\left(-m_{1}^{2}+3 m_{1}-3\right) p^{2}+\left(2 m_{2} m_{1}-3 m_{2}-1\right) p-m_{2}^{2}=0
$$

has a non-zero solution $p$. With this $p$, we see that a corresponding rational function is

$$
R(z)=\frac{-\left(\left(m_{1}-2\right) p-m_{2}\right)\left(\left(m_{1} p+1\right) z+\left(m_{1}^{2}-2 m_{1}\right) p-m_{2} m_{1}\right) z}{p\left(\left(m_{1}-1\right) p-m_{2}+1\right)\left(z^{2}-z+1\right)} .
$$

Here, $\left(m_{1}-1\right) p-m_{2}+1 \neq 0$ from the assumption, and we conclude the assertion when $d=2$.

Note that, in terms of the fundamental symmetric functions,

$$
\sigma_{1}=m_{1}+m_{2}+m_{3}, \quad \sigma_{2}=m_{1} m_{2}+m_{1} m_{3}+m_{2} m_{3}, \quad \text { and } \quad \sigma_{3}=m_{1} m_{2} m_{3},
$$

the natural projection of $N_{2} \cong\left\{\left(a_{2}, a_{1}\right) \mid a_{2}^{2}+a_{1} a_{2}+a_{1}^{2} \neq 0\right\}$ to $\mathrm{M}_{2}$ is given by

$$
\begin{aligned}
\sigma_{1} & =\frac{2 a_{2}^{2}+a_{1}^{2} a_{2}+a_{1}^{3}-2 a_{1}^{2}+3 a_{1}}{a_{2}^{2}+a_{1} a_{2}+a_{1}^{2}}, \\
\sigma_{2} & =\frac{-\left(a_{1}^{2}-2 a_{1}\right) a_{2}^{2}+\left(a_{1}-2\right) a_{2}-2 a_{1}^{3}+4 a_{1}^{2}-4 a_{1}+3}{a_{2}^{2}+a_{1} a_{2}+a_{1}^{2}}, \\
\sigma_{3} & =\sigma_{1}-2 .
\end{aligned}
$$


In general, we obtain the following.

Theorem 2.4. For every $d \geq 2$, the natural projection of $N_{d}$ to $\mathrm{M}_{d}$ is surjective.

Proof. The assertion for the case that $d=2$ is shown in the above example. When $d=3$, we can show the assertion by direct calculations using a symbolic and algebraic computation system, the detail of which is contained in $\S 4$ for the sake of the readers' convenience. So, we assume that $d \geq 4$ in the sequel of the proof.

Let $x$ be a point of $\mathrm{M}_{d}$ and $R(z)$ a rational function of degree $d$ contained in the Möbius conjugacy class $x$. Then we may assume that $R(z)$ is canonical and $R(0)=0$, by taking a Möbius conjugate of $R(z)$ if necessary, which implies in particular that

$$
a_{0}=0 \quad \text { and } \quad b_{0} \neq 0
$$

Next, if we take conjugate of $R(z)$ by a translation $L(z)=z+\alpha$, then we have

$$
L^{-1} \circ R \circ L(z)=\frac{\left(a_{d}(z+\alpha)^{d}+\cdots+a_{0}\right)-\alpha\left((z+\alpha)^{d}+\cdots+b_{0}\right)}{(z+\alpha)^{d}+\cdots+b_{0}},
$$

which we write as

$$
\frac{\tilde{a}_{d} z^{d}+\cdots+\tilde{a}_{0}}{z^{d}+\tilde{b}_{d-1} z^{d-1}+\cdots+\tilde{b}_{0}} .
$$

Here, if $\alpha$ is a fixed point of $R(z)$, then

$$
\tilde{a}_{0}=0, \quad \text { and } \quad \tilde{b}_{0} \neq 0 .
$$

Also, taking as $\alpha$ one of fixed points of $R(z)$ with the largest multiplicities, we may assume that $R(z)$ has no non-zero fixed points with multiplicity $d$. Moreover, if 0 is a non-simple fixed point of $L^{-1} \circ R \circ L(z)$, then

$$
\tilde{a}_{1}=\tilde{b}_{0} \text {. }
$$

And hence if $\tilde{a}_{1} \neq \tilde{b}_{0}$, then every fixed point of $R(z)$ is simple, and there is a nonzero fixed point $\zeta_{R}$ of $R(z)$ such that $\tilde{b}_{1} \neq 0$. Indeed, letting $\left\{\zeta_{1}, \cdots, \zeta_{d}\right\}$ be the set of non-zero fixed points of $R(z)$, we consider conjugates of $R(z)$ by $L_{k}(z)=z+\zeta_{k}$. Then

$$
\tilde{b}_{1}=d \zeta_{k}^{d-1}+(d-1) b_{d-1} \zeta_{k}^{d-2}+\cdots+b_{1}
$$

cannot be 0 for all $k$. Also, repeating such a change of fixed points again, if necessary, we can further assume that there are neither circles nor lines in $\mathbb{C}-\{0\}$, which contain all non-zero fixed points, since we have assumed that $d \geq 4$.

Thus we may assume from the beginning that $a_{0}=0, b_{0} \neq 0$, and $\left(d b_{0}-a_{1}\right) z+b_{1}$ is not constantly $0, R(z)$ has no non-zero fixed points with the multiplicities $d$, and if $R(z)$ has simple fixed points only, then there are neither circles nor lines in $\mathbb{C}-\{0\}$ which contain all non-zero fixed points.

Now, set

Then we have

$$
T(z)=\frac{z}{p z+q} \quad(q \neq 0)
$$

$$
T^{-1} \circ R \circ T(z)=\frac{q\left(a_{d} z^{d}+\cdots+a_{1} z(p z+q)^{d-1}\right)}{-p\left(a_{d} z^{d}+\cdots+a_{1} z(p z+q)^{d-1}\right)+\left(z^{d}+\cdots+b_{0}(p z+q)^{d}\right)} .
$$

The constant term of the numerator remains 0 , and the coefficients of $z^{d}$ in the numerator and the denominator change to

$$
a_{d}^{*}(p, q)=q\left(a_{d}+\cdots+a_{1} p^{d-1}\right)
$$


and

$$
b_{d}^{*}(p)=-p\left(a_{d}+\cdots+a_{1} p^{d-1}\right)+\left(1+\cdots+b_{0} p^{d}\right),
$$

respectively. If $b_{d}^{*}(p) \neq 0$, divide both the numerator and the denominator of the conjugate $T^{-1} \circ R \circ T(z)$ by $b_{d}^{*}(p)$. Then the coefficients $a_{1}, b_{0}$ and $b_{1}$, for instance, change to

$$
\begin{aligned}
& a_{1}(p, q)=\frac{a_{1} q^{d}}{b_{d}^{*}(p)}, \quad b_{0}(p, q)=\frac{b_{0} q^{d}}{b_{d}^{*}(p)}, \\
& b_{1}(p, q)=\frac{-a_{1} p q^{d-1}+b_{1} q^{d-1}+d b_{0} p q^{d-1}}{b_{d}^{*}(p)} .
\end{aligned}
$$

Also, the condition $b_{1}(p, q) / b_{0}(p, q)=-1$ implies that

$$
q=q(p)=-\frac{\left(d b_{0}-a_{1}\right) p+b_{1}}{b_{0}} .
$$

First, if $b_{0}=a_{1}$, then $b_{0}(p, q(p))$ is a rational function of $p$ such that the degrees of the numerator and the denominator are exactly $d$ and not greater than $d-1$, respectively. Hence there is a finite $p$ with $b_{0}(p, q(p))=1$. Next, if $d b_{0}=a_{1}$, then $b_{0}(p, q(p))$ is a rational function of $p$ such that the degree of the denominator is exactly $d$ and the numerator is a non-zero constant. Hence there is a finite $p$ with $b_{0}(p, q(p))=1$. Finally, if otherwise, namely if $b_{0} \neq a_{1}$ and $d b_{0} \neq a_{1}$, then the degrees of the numerator and the denominator are exactly $d$ and $R(z)$ has simple fixed points only. We write non-zero fixed points of $R(z)$ as $\left\{\zeta_{k}\right\}_{k=1}^{d}$. Suppose that $b_{0}(p, q(p))$ can take the value 1 at $\infty$ only. Then with some non-zero constant $C$,

$$
b_{0}(p, q(p))=1+\frac{C}{b_{d}^{*}(p)},
$$

which implies that $\left\{1 / \zeta_{k}\right\}_{k=1}^{d}$ lie on the same circle, for $b_{d}^{*}(p)=\prod_{k=1}^{d}\left(1-\zeta_{k} p\right)$. But then, $\left\{\zeta_{k}\right\}_{k=1}^{d}$ should be on a same circle or a line not containing 0 , which contradicts one of the assumptions from the beginning. Hence we also conclude in this case that there is a finite $p$ such that $b_{0}(p, q(p))=1$.

Thus we obtain a $T(z)$ such that $T^{-1} \circ R \circ T(z)$ belongs to $N_{d}$ if $d \geq 4$, and the proof is now complete.

For a generic point of $\mathrm{M}_{d}$, there are only a finite number of rational functions in $N_{d}$ belonging to the point, as is seen from the proof of Theorem 2.4. On the other hand, some points of $\mathrm{M}_{d}$ can blow up in $N_{d}$ as in the following example.

Example 2.5. Set

$$
R(z)=\frac{-3 z^{3}-4 z^{2}-2 z}{z^{3}-z-1}
$$

Then $R(z)$ has a simple fixed point at 0 , and one with multiplicity 3 at -1 .

As in the proof of Theorem 2.4, letting

$$
T(z)=\frac{z}{p z-1-p} \quad(p \neq-1)
$$

set $R_{p}(z)=T^{-1} \circ R \circ T(z)$. Then we have

$$
R_{p}(z)=\frac{\left(2 p^{2}+4 p+3\right) z^{3}+\left(-4 p^{2}-8 p-4\right) z^{2}+\left(2 p^{2}+4 p+2\right) z}{\left(p^{2}+2 p+1\right) z^{3}+\left(-p^{2}-2 p\right) z^{2}+\left(-p^{2}-2 p-1\right) z+p^{2}+2 p+1} .
$$


Hence if we set $\tilde{p}=1 /\left(p^{2}+2 p+1\right)$,

$$
R_{p}(z)=\tilde{R}_{\tilde{p}}(z)=\frac{(\tilde{p}+2) z^{3}-4 z^{2}+2 z}{z^{3}+(\tilde{p}-1) z^{2}-z+1} .
$$

Thus $\tilde{R}_{\tilde{p}}(z)$ belongs to $N_{3}$, and represents the same point of $\mathrm{M}_{3}$ for every non-zero $\tilde{p}$. Indeed, every $\tilde{R}_{\tilde{p}}(z)$ is conjugate to $\tilde{R}_{1}(z)$ by

$$
S(z)=\frac{z}{\left(1-\tilde{p}^{-1 / 2}\right) z+\tilde{p}^{-1 / 2}} .
$$

\section{A stratification OF THE MOdUli SPACE}

Every rational function $R(z)=P(z) / Q(z)$ of degree $d$ not fixing $\infty$ can also be written as

$$
R(z)=z-\frac{\hat{P}(z)}{Q(z)}
$$

with monic polynomials $\hat{P}(z)$ and $Q(z)$ of degree $d+1$ and $d$, respectively. Using this representation, we have another system of parameters, some of which are fixed points of $R(z)$.

Definition 3.1. Let

$$
R(z)=z-\frac{\hat{P}(z)}{Q(z)}
$$

with

$$
\hat{P}(z)=z Q(z)-P(z)=\prod_{j=1}^{p}\left(z-\zeta_{j}\right)^{n_{j}} \quad\left(\zeta_{j} \in \mathbb{C}\right),
$$

where $\zeta_{j}$ are mutually distinct and $n_{j}$ are positive integers which satisfy

$$
\sum_{k=1}^{p} n_{k}=d+1
$$

Then we call the set $\left\{n_{1}, \cdots, n_{p}\right\}$ the overlap type of fixed points of $R(z)$.

We set

$$
C\left\{n_{1}, \cdots, n_{p}\right\}=\left\{R(z) \in C_{d} \mid \text { the overlap type is }\left\{n_{1}, \cdots, n_{p}\right\}\right\}
$$

and call it the $\left\{n_{1}, \cdots, n_{p}\right\}$-locus of $C_{d}$. The subset

$$
C_{d}^{\prime}=\left\{R(z) \in C_{d} \mid \text { the overlap type is not }\{1, \cdots, 1\}\right\}
$$

of $C_{d}$ is called the overlap locus of $C_{d}$.

Similarly, we can define the $\left\{n_{1}, \cdots, n_{p}\right\}$-locus of $N_{d}$ by setting

$$
N\left\{n_{1}, \cdots, n_{p}\right\}=\left\{R(z) \in N_{d} \mid \text { the overlap type is }\left\{n_{1}, \cdots, n_{p}\right\}\right\} .
$$

Also, the subset

$$
N_{d}^{\prime}=\left\{R(z) \in N_{d} \mid \text { the overlap type is not }\{1, \cdots, 1\}\right\}
$$

of $N_{d}$ is called the overlap locus of $N_{d}$. 
Since the overlap type of fixed points is invariant under Möbius conjugation, Theorem 2.4 implies the following result.

Corollary 3.2. Let $\mathrm{M}_{d}^{\prime}$ be the subset of all points of $\mathrm{M}_{d}$ represented by rational functions having non-simple fixed points. Then the natural projection $\pi$ of $N_{d}^{\prime}$ to $\mathrm{M}_{d}^{\prime}$ is surjective for every $d \geq 2$.

Definition 3.3. The image of every $\left\{n_{1}, \cdots, n_{p}\right\}$-locus of $N_{d}$ by $\pi$ is called the $\left\{n_{1}, \cdots, n_{p}\right\}$-stratum of $\mathrm{M}_{d}$, and is denoted by $M\left\{n_{1}, \cdots, n_{p}\right\}$. The resulting stratification of $\mathrm{M}_{d}$ is called the overlap type stratification.

Remark 3.4. The above loci are defined by algebraic equations (cf. Examples 3.5 and 3.6) and hence Zariski open subsets of complex algebraic sets (with respect to the system of coefficient parameters). For instance,

$$
C_{d}^{\prime}=\left\{\frac{\hat{P}(z)}{Q(z)} \in C_{d} \mid \operatorname{Discr}(\hat{P})=0\right\} .
$$

Example 3.5. In the case of $d=2$, under the resultant condition

$$
\begin{aligned}
& C\{3\} \cong\left\{\left(a_{2}, a_{1}, a_{0}, b_{1}, b_{0}\right)\right.\left.\begin{array}{l}
a_{1}=b_{0}-\left(b_{1}-a_{2}\right)^{2} / 3, \\
a_{0}=-\left(b_{1}-a_{2}\right)^{3} / 27
\end{array}\right\}, \\
& C_{2}^{\prime} \cong\left\{\left(a_{2}, a_{1}, a_{0}, b_{1}, b_{0}\right) \mid \begin{array}{l}
-27 a_{0}^{2}+a_{0}\left\{4\left(b_{1}-a_{2}\right)^{3}-18\left(b_{0}-a_{1}\right)\left(b_{1}-a_{2}\right)\right\} \\
+\left(a_{1}-b_{0}\right)^{2}\left(b_{1}-a_{2}\right)^{2}+4\left(a_{1}-b_{0}\right)^{3}=0
\end{array}\right\}, \\
& N\{3\} \cong\{(-1,1,0,-1,1)\}, \\
& N_{2}^{\prime} \cong\left\{\left(a_{2}, a_{1}, 0,-1,1\right) \mid a_{1}-1=-\left(a_{2}+1\right)^{2} / 4 \text { or } \quad a_{1}=1\right\} .
\end{aligned}
$$

Example 3.6. In the case of $d=3$, under the resultant condition

$$
\begin{aligned}
& C\{4\} \cong\left\{\left(a_{3}, a_{2}, a_{1}, a_{0}, b_{2}, b_{1}, b_{0}\right)\right.\left.\begin{array}{l}
a_{2}=b_{1}-3\left(b_{2}-a_{3}\right)^{2} / 8, \quad a_{1}=b_{0}-\left(b_{2}-a_{3}\right)^{3} / 16, \\
a_{0}=-\left(b_{2}-a_{3}\right)^{4} / 256
\end{array}\right\}, \\
& C_{3}^{\prime} \cong\left\{\left(a_{3}, a_{2}, a_{1}, a_{0}, b_{2}, b_{1}, b_{0}\right) \mid \mathrm{D}=0\right\},
\end{aligned}
$$

where

$$
\begin{gathered}
\mathrm{D}=256 a_{0}^{3}+a_{0}^{2}\left\{128\left(b_{1}-a_{2}\right)^{2}-144\left(b_{2}-a_{3}\right)^{2}\left(b_{1}-a_{2}\right)+27\left(b_{2}-a_{3}\right)^{4}\right. \\
\left.+192\left(b_{2}-a_{3}\right)\left(b_{0}-a_{1}\right)\right\}+a_{0}\left\{16\left(b_{1}-a_{2}\right)^{4}-4\left(b_{2}-a_{3}\right)^{2}\left(b_{1}-a_{2}\right)^{3}\right. \\
-80\left(b_{0}-a_{1}\right)\left(b_{2}-a_{3}\right)\left(b_{1}-a_{2}\right)^{2}+18\left(b_{0}-a_{1}\right)\left(\left(b_{2}-a_{3}\right)^{3}+8\left(b_{0}-a_{1}\right)\right)\left(b_{1}-a_{2}\right) \\
\left.-6\left(b_{0}-a_{1}\right)^{2}\left(b_{2}-a_{3}\right)^{2}\right\}+\left(b_{0}-a_{1}\right)^{2}\left(4\left(b_{1}-a_{2}\right)^{3}-\left(b_{2}-a_{3}\right)^{2}\left(b_{1}-a_{2}\right)^{2}\right. \\
\left.-18\left(b_{0}-a_{1}\right)\left(b_{2}-a_{3}\right)\left(b_{1}-a_{2}\right)+\left(b_{0}-a_{1}\right)\left(4\left(b_{2}-a_{3}\right)^{3}+27\left(b_{0}-a_{1}\right)\right)\right), \\
N\{4\} \cong\{(c,-1,1,0, c,-1,1) \mid c \in \mathbb{C}\},
\end{gathered}
$$


and

$$
\begin{aligned}
& N_{3}^{\prime} \cong\left\{\left(a_{3}, a_{2}, a_{1}, 0, b_{2},-1,1\right)\right. \\
& \left.\mid \begin{array}{c}
-27\left(a_{1}-1\right)^{2}+\left(a_{1}-1\right)\left(4\left(b_{2}-a_{3}\right)^{3}+18\left(a_{2}+1\right)\left(b_{2}-a_{3}\right)\right) \\
+\left(a_{2}+1\right)^{2}\left(b_{2}-a_{3}\right)^{2}+4\left(a_{2}+1\right)^{3}=0 \text { or } a_{1}=1
\end{array}\right\} .
\end{aligned}
$$

On the other hand, it is well known that the denominator $Q(z)$ of $R(z)$ in $C\left\{n_{1}, \cdots, n_{p}\right\}$ can be represented uniquely as

$$
Q(z)=\sum_{k=1}^{p}\left\{\left(\sum_{n=0}^{n_{k}-1} \alpha_{k, n_{k}-n}\left(z-\zeta_{k}\right)^{n}\right) \prod_{j \neq k}\left(z-\zeta_{j}\right)^{n_{j}}\right\} .
$$

In other words, $Q(z) / \hat{P}(z)$ has a unique partial fraction decomposition

$$
\frac{\alpha_{1, n_{1}}}{\left(z-\zeta_{1}\right)^{n_{1}}}+\cdots+\frac{\alpha_{1,1}}{z-\zeta_{1}}+\frac{\alpha_{2, n_{2}}}{\left(z-\zeta_{2}\right)^{n_{2}}}+\cdots+\frac{\alpha_{p, 1}}{z-\zeta_{p}}
$$

Here, the assumptions imply that $\alpha_{k, n_{k}} \neq 0$ for every $k$ and

$$
\sum_{k=1}^{p} \alpha_{k, 1}=1
$$

Definition 3.7. The set $\left\{\zeta_{k}\right\}$ of fixed points and the set $\left\{\alpha_{k, \ell}\right\}$ of coefficients give a system of parameters for $C\left\{n_{1}, \cdots, n_{p}\right\}$, and is called the system of decomposition parameters for $C\left\{n_{1}, \cdots, n_{p}\right\}$.

Theorem 3.8. Set

$$
\begin{aligned}
\tilde{E}\left\{n_{1}, \cdots, n_{p}\right\}=\{ & \left(\zeta_{1}, \cdots, \zeta_{p}, \alpha_{1,1}, \cdots, \alpha_{1, n_{1}}, \alpha_{2,1}, \cdots, \alpha_{p, n_{p}}\right) \in \mathbb{C}^{d+p+1} \\
& \left.\mid \sum_{k=1}^{p} \alpha_{k, 1}=1, \quad \alpha_{k, n_{k}} \neq 0 \quad(k=1, \cdots, p)\right\} .
\end{aligned}
$$

Then the natural projection $\Pi$ of $\tilde{E}\left\{n_{1}, \cdots, n_{p}\right\}$ to $C\left\{n_{1}, \cdots, n_{p}\right\}$ (with respect to the system of coefficient parameters) is a holomorphic surjection.

Moreover, $C\left\{n_{1}, \cdots, n_{p}\right\}$ has a complex manifold structure such that $\Pi$ is a finite-sheeted holomorphic covering projection. 
We call $\tilde{E}\left\{n_{1}, \cdots, n_{p}\right\}$ the marked $\left\{n_{1}, \cdots, n_{p}\right\}$-parameter domain.

Proof. Since $\Pi$ is a polynomial map, it is holomorphic. To show other assertions, note that the defining domains of the system of decomposition parameters is the product space

$$
\prod_{n=1}^{d+1} C_{N_{n}}\left(\mathbb{C}^{n+1}\right),
$$

where $C_{m}\left(\mathbb{C}^{n}\right)$ is the configuration space of $m$ distinct vectors in $\mathbb{C}^{n}$ and $N_{n}$ is the number of $\ell$ with $n_{\ell}=n$. In particular, $N_{n}>0$ only if

$$
\min \left\{n_{1}, \cdots, n_{p}\right\} \leq n \leq \max \left\{n_{1}, \cdots, n_{p}\right\}
$$

the set $\left\{(n, 1), \cdots,\left(n, N_{n}\right)\right\}$ is empty if there are no $\ell$ with $n_{\ell}=n$, and

$$
\sum_{n=1}^{d+1} n N_{n}=d+1
$$

The coordinates of the product space can be written explicitly as follows;

$$
\begin{aligned}
& E\left\{n_{1}, \cdots, n_{p}\right\} \\
& =\left\{\left(\left\{\left(\zeta_{1,1}, \alpha_{(1,1), 1}\right), \cdots,\left(\zeta_{1, N_{1}}, \alpha_{\left(1, N_{1}\right), 1}\right)\right\}, \cdots,\right.\right. \\
& \left\{\left(\zeta_{d+1,1}, \alpha_{(d+1,1), 1}, \cdots, \alpha_{(d+1,1), d+1}\right), \cdots,\right. \\
& \left.\left.\quad\left(\zeta_{d+1, N_{d+1}}, \alpha_{\left(d+1, N_{d+1}\right), 1}, \cdots, \alpha_{\left(d+1, N_{d+1}\right), d+1}\right)\right\}\right) \in \prod_{n=1}^{d+1} C_{N_{n}}\left(\mathbb{C}^{n+1}\right) \\
& \left.\mid \sum_{k=1}^{d+1}\left(\sum_{j=1}^{N_{k}} \alpha_{(k, j), 1}\right)=1, \quad \alpha_{(k, *), k} \neq 0 \quad(k=1, \cdots, p)\right\},
\end{aligned}
$$

where all $\zeta$ are mutually disjoint as before.

Now the map $\Pi$ is factored through by the canonical finite-sheeted holomorphic covering projection $\sigma$ of $\tilde{E}\left\{n_{1}, \cdots, n_{p}\right\}$ to $E\left\{n_{1}, \cdots, n_{p}\right\}$ and the natural holomorphic bijection $\iota$ of $E\left\{n_{1}, \cdots, n_{p}\right\}$ to $C\left\{n_{1}, \cdots, n_{p}\right\}$ :

$$
\Pi=\iota \circ \sigma .
$$

In particular, $\iota$ induces the desired complex manifold structure on $C\left\{n_{1}, \cdots, n_{p}\right\}$.

Remark 3.9. On the non-overlap locus $C\{1, \cdots, 1\}=C_{d}-C_{d}^{\prime},\left\{\alpha_{k, 1}\right\}_{k=1}^{d+1}$ in the system of decomposition parameters are nothing but the indices at the fixed points $\left\{\zeta_{k}\right\}_{k=1}^{d+1}$, which implies the assertion of Problem 12-d in [7].

Corollary 3.10. If the location and the overlap type of fixed points and the indices at them are fixed, then the resulting subset of $C\left\{n_{1}, \cdots, n_{p}\right\}$ has a natural complex manifold structure of dimension $d+1-p$. 
Proof. By Theorem 3.8, we need only to note that

$$
\operatorname{dim}_{\mathbb{C}} C\left\{n_{1}, \cdots, n_{p}\right\}=d+p .
$$

This corollary gives the affirmative answer to a conjecture of Milnor stated in the remark following Problem 12-d (see [7, p. 152]).

\section{The Proof of Theorem 2.4 For the CASE that $d=3$}

Even in the case that $d=3$, the arguments of the proof of Theorem 2.4 can be applied, but we cannot exclude the case that $R(z)$ has 4 simple fixed points $0, w_{1}, w_{2}, w_{3}$ such that $1 / w_{1}, 1 / w_{2}, 1 / w_{3}$ lie on the same circle. So, we will treat this case by direct calculation using a symbolic and algebraic computation system (cf. [1] and [2]).

For this purpose, let $0, w_{1}, w_{2}, w_{3}$ be the set of simple fixed points of a given $R(z)$ of degree 3 (having simple fixed points only). We may assume that the denominator has the form $z^{3}+b_{2} z^{2}+b_{1} z+b_{0}$ with $b_{0} \neq 0$ as before. Let

$$
T(z)=\frac{z}{p z+q} \quad(q \neq 0)
$$

and take the conjugate of $R(z)$ by $T(z)$. Then the coefficients $1, b_{1}$, and $b_{0}$ in the denominator $z^{3}+b_{2} z^{2}+b_{1} z+b_{0}$ change to

$$
\begin{gathered}
b_{3}^{*}(p)=w_{3} w_{2} w_{1} p^{3}-\left(\left(w_{2}+w_{3}\right) w_{1}+w_{3} w_{2}\right) p^{2}+\left(w_{1}+w_{2}+w_{3}\right) p-1, \\
b_{1}^{*}(p, q)=\left(w_{3} w_{2} w_{1}-2 b_{0}\right) q^{2} p-b_{1} q^{2}
\end{gathered}
$$

and

$$
b_{0}^{*}(q)=-b_{0} q^{3} .
$$

So the condition $b_{1}^{*}(p, q) / b_{0}^{*}(q)=-1$ implies that

$$
q=\frac{\left(w_{3} w_{2} w_{1}-2 b_{0}\right) p-b_{1}}{b_{0}}
$$

and the condition $b_{0}^{*}(p, q) / b_{3}^{*}(p)=1$ is the equation

$$
\begin{aligned}
& \left(-w_{1}^{3} w_{2}^{3} w_{3}^{3}+6 b_{0} w_{1}^{2} w_{2}^{2} w_{3}^{2}-13 b_{0}^{2} w_{1} w_{2} w_{3}+8 b_{0}^{3}\right) p^{3} \\
& +\left(\left(3 w_{1}^{2} w_{2}^{2} w_{3}^{2}-12 b_{0} w_{1} w_{2} w_{3}+12 b_{0}^{2}\right) b_{1}+\left(b_{0}^{2} w_{2}+b_{0}^{2} w_{1}\right) w_{3}+b_{0}^{2} w_{1} w_{2}\right) p^{2} \\
& \quad+\left(\left(-3 w_{1} w_{2} w_{3}+6 b_{0}\right) b_{1}^{2}-b_{0}^{2} w_{3}-b_{0}^{2} w_{2}-b_{0}^{2} w_{1}\right) p+b_{1}^{3}+b_{0}^{2}=0
\end{aligned}
$$

which we write as $A_{3} p^{3}+A_{2} p^{2}+A_{1} p+A_{0}=0$, where $A_{k}$ are functions of $w_{1}, w_{2}, w_{3}, b_{0}, b_{1}$. 
Here, we consider the equations

$$
A_{3}=A_{2}=A_{1}=0 .
$$

By computing the Gröbner basis of lexicographic order $b_{1}>b_{0}>w_{1}>w_{2}>w_{3}$, we obtain the conditions

$$
w_{3}=0, w_{2}=0, w_{1}=0
$$

Or

$$
W=\left(w_{2}^{2}-w_{1} w_{2}+w_{1}^{2}\right) w_{3}^{2}+\left(-w_{1} w_{2}^{2}-w_{1}^{2} w_{2}\right) w_{3}+w_{1}^{2} w_{2}^{2}=0
$$

in $\mathbb{C}\left[w_{1}, w_{2}, w_{3}\right]$. The conditions $w_{k}=0(k=1,2,3)$ contradict the assumption that $R(z)$ has 4 simple fixed points. Also, we recall that the case $W=0$ is one excluded in the proof of Theorem 2.4, and actually the condition $W=0$ implies that $1 / w_{1}, 1 / w_{2}$, and $1 / w_{3}$ form a regular triangle in $\mathbb{C}$. (If $d \geq 4$, we can assume that there are neither circles nor lines in $\mathbb{C}-\{0\}$ which contain all non-zero fixed points.)

As before, we consider the conjugate of $R(z)$ by the translation $L_{k}(z)=z+w_{k}$ for every $k$. Here we need to consider the case of $L_{1}(z)=z+w_{1}$ only, for the other cases are similar. Firstly, take the conjugate of $R(z)$ by $L_{1}(z)$, and secondly take the conjugate by $T(z)$, and we see that $R(z)$ changes to

$$
R^{\#}(z)=\frac{P^{\#}(z)}{Q^{\#}(z)}
$$

with

$$
Q^{\#}(z)=b_{3}^{\#} z^{3}+b_{2}^{\#} z^{2}+b_{1}^{\#} z+b_{0}^{\#},
$$

where

$$
\begin{aligned}
b_{3}^{\#}=\left(w_{1}^{3}+\left(-w_{2}-w_{3}\right) w_{1}^{2}+w_{3} w_{2} w_{1}\right) p^{3}+\left(3 w_{1}^{2}+\left(-2 w_{2}-2 w_{3}\right) w_{1}\right. \\
\left.+w_{3} w_{2}\right) p^{2}+\left(3 w_{1}-w_{2}-w_{3}\right) p+1 \\
b_{1}^{\#}=\left(2 w_{1} b_{1}+2 w_{1}^{2} b_{2}+3 w_{1}^{3}+\left(-w_{2}-w_{3}\right) w_{1}^{2}+w_{3} w_{2} w_{1}+2 b_{0}\right) q^{2} p \\
\quad+\left(b_{1}+2 w_{1} b_{2}+3 w_{1}^{2}\right) q^{2}
\end{aligned}
$$

and

$$
b_{0}^{\#}=\left(w_{1} b_{1}+w_{1}^{2} b_{2}+w_{1}^{3}+b_{0}\right) q^{3} .
$$

Hence the condition $b_{1}^{\#} / b_{0}^{\#}=-1$ implies that

$$
\begin{aligned}
& q=\frac{-1}{w_{1} b_{1}}+w_{1}^{2} b_{2}+w_{1}^{3}+b_{0} \\
& \times\left\{\left(2 w_{1} b_{1}+2 w_{1}^{2} b_{2}+3 w_{1}^{3}-\left(w_{2}+w_{3}\right) w_{1}^{2}+w_{3} w_{2} w_{1}+2 b_{0}\right) p\right. \\
&\left.+b_{1}+2 w_{1} b_{2}+3 w_{1}^{2}\right\},
\end{aligned}
$$

and the condition $b_{0}^{\#} / b_{3}^{\#}=1$ is the equation

$$
B_{3} p^{3}+B_{2} p^{2}+B_{1} p+B_{0}=0
$$


with

$$
\begin{aligned}
& B_{3}=-\left\{w_{1} b_{1}+w_{1}^{2} b_{2}+2 w_{1}^{3}+\left(-w_{2}-w_{3}\right) w_{1}^{2}+w_{3} w_{2} w_{1}+b_{0}\right\} \\
& \times\left\{8 w_{1}^{2} b_{1}^{2}+\left(16 w_{1}^{3} b_{2}+21 w_{1}^{4}+\left(-5 w_{2}-5 w_{3}\right) w_{1}^{3}+5 w_{3} w_{2} w_{1}^{2}+16 b_{0} w_{1}\right) b_{1}\right. \\
& +8 w_{1}^{4} b_{2}^{2}+\left(21 w_{1}^{5}+\left(-5 w_{2}-5 w_{3}\right) w_{1}^{4}+5 w_{3} w_{2} w_{1}^{3}+16 b_{0} w_{1}^{2}\right) b_{2} \\
& +14 w_{1}^{6}+\left(-7 w_{2}-7 w_{3}\right) w_{1}^{5}+\left(w_{2}^{2}+9 w_{3} w_{2}+w_{3}^{2}\right) w_{1}^{4} \\
& +\left(-2 w_{3} w_{2}^{2}-2 w_{3}^{2} w_{2}+21 b_{0}\right) w_{1}^{3}+\left(w_{3}^{2} w_{2}^{2}-5 b_{0} w_{2}-5 b_{0} w_{3}\right) w_{1}^{2} \\
& \left.+5 b_{0} w_{3} w_{2} w_{1}+8 b_{0}^{2}\right\} \\
& B_{2}=\left(-12 w_{1}^{2} b_{1}^{3}-\left(48 w_{1}^{3} b_{2}+75 w_{1}^{4}-\left(14 w_{2}+14 w_{3}\right) w_{1}^{3}+13 w_{3} w_{2} w_{1}^{2}+24 b_{0} w_{1}\right) b_{1}^{2}\right. \\
& +\left(-60 w_{1}^{4} b_{2}^{2}+\left(-186 w_{1}^{5}+\left(40 w_{2}+40 w_{3}\right) w_{1}^{4}-38 w_{3} w_{2} w_{1}^{3}-72 b_{0} w_{1}^{2}\right) b_{2}\right. \\
& -141 w_{1}^{6}+\left(58 w_{2}+58 w_{3}\right) w_{1}^{5}+\left(-3 w_{2}^{2}-62 w_{3} w_{2}-3 w_{3}^{2}\right) w_{1}^{4} \\
& +\left(6 w_{3} w_{2}^{2}+6 w_{3}^{2} w_{2}-114 b_{0}\right) w_{1}^{3}+\left(-3 w_{3}^{2} w_{2}^{2}+16 b_{0} w_{2}+16 b_{0} w_{3}\right) w_{1}^{2} \\
& \left.-14 b_{0} w_{3} w_{2} w_{1}-12 b_{0}^{2}\right) b_{1}-24 w_{1}^{5} b_{2}^{3}+\left(-111 w_{1}^{6}+\left(26 w_{2}+26 w_{3}\right) w_{1}^{5}\right. \\
& \left.-25 w_{3} w_{2} w_{1}^{4}-48 b_{0} w_{1}^{3}\right) b_{2}^{2}+\left(-168 w_{1}^{7}+\left(76 w_{2}+76 w_{3}\right) w_{1}^{6}\right. \\
& +\left(-6 w_{2}^{2}-86 w_{3} w_{2}-6 w_{3}^{2}\right) w_{1}^{5}+\left(12 w_{3} w_{2}^{2}+12 w_{3}^{2} w_{2}-150 b_{0}\right) w_{1}^{4} \\
& \left.+\left(-6 w_{3}^{2} w_{2}^{2}+28 b_{0} w_{2}+28 b_{0} w_{3}\right) w_{1}^{3}-26 b_{0} w_{3} w_{2} w_{1}^{2}-24 b_{0}^{2} w_{1}\right) b_{2} \\
& -84 w_{1}^{8}+\left(56 w_{2}+56 w_{3}\right) w_{1}^{7}+\left(-9 w_{2}^{2}-73 w_{3} w_{2}-9 w_{3}^{2}\right) w_{1}^{6} \\
& +\left(18 w_{3} w_{2}^{2}+18 w_{3}^{2} w_{2}-114 b_{0}\right) w_{1}^{5}+\left(-9 w_{3}^{2} w_{2}^{2}+40 b_{0} w_{2}+40 b_{0} w_{3}\right) w_{1}^{4} \\
& \left.-38 b_{0} w_{3} w_{2} w_{1}^{3}-39 b_{0}^{2} w_{1}^{2}+\left(2 b_{0}^{2} w_{2}+2 b_{0}^{2} w_{3}\right) w_{1}-b_{0}^{2} w_{3} w_{2}\right), \\
& B_{1}=\left(-6 w_{1} b_{1}^{3}+\left(-30 w_{1}^{2} b_{2}-48 w_{1}^{3}+\left(4 w_{2}+4 w_{3}\right) w_{1}^{2}-3 w_{3} w_{2} w_{1}-6 b_{0}\right) b_{1}^{2}\right. \\
& +\left(-48 w_{1}^{3} b_{2}^{2}+\left(-150 w_{1}^{4}+\left(14 w_{2}+14 w_{3}\right) w_{1}^{3}-12 w_{3} w_{2} w_{1}^{2}-24 b_{0} w_{1}\right) b_{2}\right. \\
& \left.-114 w_{1}^{5}+\left(20 w_{2}+20 w_{3}\right) w_{1}^{4}-18 w_{3} w_{2} w_{1}^{3}-42 b_{0} w_{1}^{2}+\left(2 b_{0} w_{2}+2 b_{0} w_{3}\right) w_{1}\right) b_{1} \\
& -24 w_{1}^{4} b_{2}^{3}+\left(-111 w_{1}^{5}+\left(13 w_{2}+13 w_{3}\right) w_{1}^{4}-12 w_{3} w_{2} w_{1}^{3}-24 b_{0} w_{1}^{2}\right) b_{2}^{2} \\
& +\left(-168 w_{1}^{6}+\left(38 w_{2}+38 w_{3}\right) w_{1}^{5}-36 w_{3} w_{2} w_{1}^{4}-78 b_{0} w_{1}^{3}\right. \\
& \left.+\left(2 b_{0} w_{2}+2 b_{0} w_{3}\right) w_{1}^{2}\right) b_{2}-84 w_{1}^{7}+\left(28 w_{2}+28 w_{3}\right) w_{1}^{6}-27 w_{3} w_{2} w_{1}^{5} \\
& \left.-60 b_{0} w_{1}^{4}+\left(2 b_{0} w_{2}+2 b_{0} w_{3}\right) w_{1}^{3}-3 b_{0}^{2} w_{1}+b_{0}^{2} w_{2}+b_{0}^{2} w_{3}\right),
\end{aligned}
$$

and

$$
\begin{aligned}
B_{0}= & -b_{1}^{3}+\left(-6 w_{1} b_{2}-10 w_{1}^{2}\right) b_{1}^{2}+\left(-12 w_{1}^{2} b_{2}^{2}-38 w_{1}^{3} b_{2}-29 w_{1}^{4}-2 b_{0} w_{1}\right) b_{1} \\
& -8 w_{1}^{3} b_{2}^{3}-37 w_{1}^{4} b_{2}^{2}+\left(-56 w_{1}^{5}-2 b_{0} w_{1}^{2}\right) b_{2}-28 w_{1}^{6}-2 b_{0} w_{1}^{3}-b_{0}^{2} .
\end{aligned}
$$

Now, we consider the equations

$$
A_{3}=A_{2}=A_{1}=0 \text { and } B_{3}=B_{2}=B_{1}=0 .
$$


By computing the Gröbner basis as before, we obtain the conditions

$$
w_{3}=0, w_{2}=0, \text { or } w_{2}-w_{3}=0,
$$

in $\mathbb{C}\left[w_{2}, w_{3}\right]$, which again gives a contradiction to the assumption. Therefore, either equations (4.1) or (4.2) has a solution $p$.

Thus we have shown the assertion of Theorem 2.4 for the case that $d=3$.

\section{REFERENCES}

1. D. Cox, J. Little, and D. O'Shea, Ideals, varieties, and algorithms, UTM, Springer-Verlag, 1998.

2. D. Cox, J. Little, and D. O'Shea, Using Algebraic Geometry, GTM 185, Springer-Verlag, 1998. MR:1639811 (99h:13033)

3. M. Fujimura, The moduli space of rational maps and surjectivity of multiplier representation, Comp. Meth. Funct. Th. 7 (2007), 345-360. MR2376676 (2008k:30049)

4. M. Fujimura and M. Taniguchi, A compactification of the moduli space of polynomials, Proc. Amer. Math. Soc. 136 (2008), 3601-3609. MR2415044 (2009d:37081)

5. C. McMullen, Families of rational maps and iterative root-finding algorithms, Ann. of Math. 125 (1987), 467-493. MR890160 (88i:58082)

6. J. Milnor, Geometry and dynamics of quadratic rational maps, Experiment. Math. 2 (1993), 37-83. MR.1246482 (96b:58094)

7. _ Dynamics in one complex variable, third ed., Princeton University Press, 2006. MR2193309 (2006g:37070)

Department of Mathematics, National Defense Academy, Yokosuka 239-8686, Japan

E-mail address: masayo@nda.ac.jp

Department of Mathematics, Nara Women's University, Nara 630-8506, Japan

E-mail address: tanig@cc.nara-wu.ac.jp 